\title{
UN MODELO PEDAGÓGICO PARA LA EDUCACIÓN AMBIENTAL DESDE LA PERSPECTIVA DE LA MODIFICABILIDAD ESTRUCTURAL COGNITIVA
}

\author{
WILLIAM R. AVENDAÑO $C^{1}$.
}

Recibido el 8 de agosto de 2012 y aprobado el 13 de septiembre de 2012

\section{RESUMEN}

El artículo tiene como objetivo formular un modelo pedagógico para la educación ambiental atendiendo a la urgencia de crear mecanismos e instrumentos que contribuyan con el desarrollo sostenible del planeta. Para ello, se abordan los principales aspectos teóricos sobre educación, pedagogía, modelos pedagógicos y corrientes de la educación ambiental. Para el diseño se ha analizado e incorporado en el modelo la teoría de la Modificabilidad Estructural Cognitiva y la Experiencia de Aprendizaje Mediado del Reueven Feuerstein. El diseño responde a las exigencias de la sociedad actual y a una perspectiva del medio ambiente desde diversos enfoques. Asimismo, plantea un rol del maestro y del educando como agentes dinamizadores de la construcción del conocimiento y la modificación de conductas responsables con el medio ambiente.

\section{PALABRAS CLAVE:}

Educación ambiental, modificabilidad estructural cognitiva, experiencia de aprendizaje mediado, modelo pedagógico, educación.

\section{A PEDAGOGICAL MODEL FOR ENVIROMENTAL EDUCATION FROM THE PERSPECTIVE OF THE COGNITIVE STRUCTURAL MODIFIABILITY}

\begin{abstract}
The objective of this article is to formulate a pedagogical model for environmental education in response to the urgent need to create mechanisms and instruments that contribute to global sustainable development. To do so, the main theoretical aspects of education, pedagogy, pedagogical models and trends in environmental education are addressed. The Structural Cognitive Modifiability and Mediated Learning Experience Reueven Feuerstein theories have been analyzed and incorporated in the design. The design responds to the demands of modern society and to an environmental perspective from different approaches. Likewise, the role of teacher and the role of the learner as revitalizing agents of knowledge construction and modification of responsible behaviors with the environment are described.
\end{abstract}




\section{KEY WORDS:}

Environmental Education, Structural Cognitive Modifiability, Mediated Learning Experience, Pedagogical Model, Education.

\section{INTRODUCCIÓN}

La educación es un proceso de tipo socio-cultural (Avendaño \& Parada, 2011) en el cual se reproducen las formas, costumbres, conocimientos, normas, conductas y paradigmas de una determinada sociedad en un tiempo histórico específico (Durkheim, 1976). Forero (2001) explica que es
"necesario potenciar el desarrollo socio-cultural de los grupos y las comunidades; responder a las necesidades relacionadas con lo macro político, la normatividad sobre la educación, la escuela y su funcionamiento, así como las disciplinas específicas que le son propias en una sociedad".

La educación constituye una de las maneras que los hombres han ideado para construir su humanidad (Wenstein, et. al. 2006), alcanzar un alto grado de bienestar y contribuir a la sana convivencia entre los sujetos. Sin embargo, la educación también ha jugado un papel en el ejercicio del poder (Thompson, 1998; Foucault, 1994) como ha sido a través del modelo tradicional establecido un par de siglos atrás por la sociedad moderna para formar sujetos con habilidades memorísticas y sumidas. Foucault (1994) enuncia que el hombre, con relación a la educación, "existe en y a través de un sistema político [en donde] el poder político proporciona cierto espacio al individuo: un espacio dónde comportarse, dónde adoptar una postura particular, sentarse de una determinada forma y trabajar continuamente".

De acuerdo con cada una de las características de las sociedades, la educación se transforma con la finalidad de responder a las exigencias que se plantean (Vázquez \& Bárcena, 2000). En este marco aparecen modelos y teorías para resolver los problemas de la educación respecto del entorno de los sujetos de aprendizaje; a pesar de las transformaciones profundas que han existido en las últimas décadas en todas las esferas (social, cultural, política, económica y tecnológica), la educación y la pedagogía han quedado rezagadas a los viejos modelos como si el mundo no se hubiese transformado.

Diversos autores abordan las temáticas de los modelos pedagógicos desde diferentes perspectivas con la finalidad de establecer ciertas relaciones que respondan a las situaciones problemicas que se originan en la cotidianidad de los educandos. De allí que no exista un modelo pedagógico único ni universal, pues cada uno asume al hombre y el papel de los 
actores educativos desde una perspectiva distinta. En efecto, los diversos autores definen, explican y clasifican los modelos pedagógicos sin existir un criterio homogéneo para dicha actividad.

Un modelo pedagógico puede ser definido como representaciones "particulares de interrelación entre los parámetros pedagógicos" (Flórez, 1999), es decir, que un modelo pedagógico determina cómo son las relaciones entre los elementos que se involucran en el proceso de enseñanzaaprendizaje: el maestro, el educando, el objeto de estudio, el entorno, etc. El objetivo de un modelo pedagógico es hacer efectivo el traspaso del conocimiento en contextos socioculturales específicos, los cuales se interrelacionan en una esfera de complejidad. Es urgente el diseño y puesta en práctica de modelos que aísle las materias y asignaturas, y se promuevan procesos de formación.

Morin (1999) explica que la educación del futuro tiene como supuestos básicos siete saberes:

- El saber centrado en los estudios del cerebro, la mente y la cultura como factores esenciales para la promoción de una educación basada en la metacognición.

- El saber enfocado hacia el desarrollo de la inteligencia en donde sea posible la ubicación de la información en contextos específicos.

- El saber que reconozca la unidad y complejidad humana en relación con las ciencias naturales, las ciencias humanas, la literatura y la filosofía.

- El saber dirigido a la complejidad de la crisis planetaria, es decir, hacia la formación de sujetos para responder a la crisis de la postmodernidad en la que están envueltos todos los seres humanos con un presente y un destino compartido.

- El saber como instrumento para asumir y enfrentar los fenómenos inesperados, en un mundo donde es imposible predecir el futuro.

- El saber como forma de mitigación de problemas sociales basados en la incomprensión como el racismo y la xenobia, que aísla a los seres humanos, amplia las diferencias y excluye grupos humanos.

- El saber para la formación de seres éticos y ciudadanos activos con conciencia política hacia la tierra y las demás personas.

En el pensamiento de Morin (1999) prevalece la complejidad como elemento fundamental de la educación y de los modelos pedagógicos, ya que expresa no sólo los tipos de saberes a transmitir, sino que determina el tipo de sociedad actual como el papel de los sujetos que intervienen en el acto educativo. Resalta de las ideas expuestas el papel del hombre en el cosmos y la importancia del mismo en la reconstrucción de su identidad en relación con la tierra. Esto es, no se puede ver al hombre desde una óptica aislada en el planeta: 
Nuestro planeta erra en el cosmos. Debemos asumir las consecuencias de esta situación marginal, periférica, que es la nuestra. Como seres vivos de este planeta, dependemos vitalmente de la biósfera terrestre; debemos reconocer nuestra muy física y muy biológica identidad terrenal (Morin, 1999).

En el espectro que ofrece Morin (1999) y otros autores como Kanitscheider (2001), Osset (2009), Nasr (1982), se evidencia una época de crisis en la relación del hombre con el medio ambiente. La inestabilidad y el desequilibrio son dos adjetivos con lo que se puede describir la relación del hombre con el medio ambiente, pues el modelo de vida que ha construido el hombre no ha atendido a los ámbitos social, cultural, económico, filosófico, estético, axiológico, etc. Frente a todas estas problemáticas, la educación tiene un desafío enorme y complejo, encontrar nuevas rutas y generar nuevos compromisos (Organización de las Naciones Unidas para la Educación, la Ciencia y la Tecnología -UNESCO- 1998).

Las causas de tal maraña de problemas ambientales tienen su origen en el despliegue económico y tecnológico (Capra, 2002; Dickson, 1978) provocado por la II Revolución Industrial, donde se estimaba que los recursos y sus fuentes eran inagotables (Ministerio de Educación-Gobierno de la ciudad de Buenos Aires, 2009; Avendaño, 2011) y que el dominio de la naturaleza con el desarrollo de la ciencia era una posibilidad (Porto, 2000).

Los esfuerzos en la creación de corrientes y modelos en el ámbito de la educación ambiental han sido numerosos como respuesta a la crisis evidente del hombre. Sauvé (2004), quien realiza un recorrido por las diversas corrientes y modelos en el campo de la educación ambiental, no deja de advertir la difícil tarea que resulta el encontrarse con una gran cantidad de discursos en los que se "proponen diversas maneras de concebir y de practicar la acción educativa" y en las que se erigen "«capillas» pedagógicas que proponen la manera «correcta» de educar, «el mejor» programa, «el» método adecuado". Cada autor, investigador o maestro tiene una representación particular pues comprenden la realidad y al hombre de una manera específica (Zeichner, 1993; Hinojosa, López \& Barroso, 1998)

En este artículo se pretende formular un modelo pedagógico para la educación ambiental que responda a las necesidades actuales del hombre en su relación con el medio ambiente con apoyo de los presupuestos ofrecidos por la teoría de la Modificabilidad Estructural Cognitiva (MEC). También se aborda, en primer lugar, el tema de modelo pedagógico y las corrientes alrededor de la educación ambiental, posteriormente la teoría de la Modificabilidad Estructural Cognitiva (MEC) y la Experiencia de Aprendizaje Mediado (EAM), y finalmente, se representa y explica el modelo pedagógico ideado para la educación ambiental. 


\section{LOS MODELOS PEDAGÓGICOS}

Son representaciones, pero dicha definición no permite identificar todos los elementos epistémicos que componen el concepto modelo pedagógico. Un modelo constituye un "pilar metateórico sobre los que se edifica las ciencias naturales" (Galagovsky \& Adúriz-Bravo, 2001) y los cuales se encuentran relacionados directamente con la teoría. Para Galagovsky y Adúriz-Bravo (2001) los modelos tienen tres características especiales:

- Los modelos son construcciones provisorias y perfectibles, es decir, que no son absolutos ni determinados, ya que pueden variar o desaparecer de acuerdo al avance de la ciencia.

- Los modelos son alternativos y pueden no coincidir entre sí, toda vez que las teorías de las que parten son distintas.

- Los modelos no desplazan en su totalidad los anteriores esquemas, porque los modelos se construyen a partir de principios y concepciones que ya han sido abordados previamente.

Entonces un modelo, en el caso de la pedagogía, no constituye una representación absoluta ni universal. Está formada a partir de diversos aportes teóricos los cuales son tomados conforme a las necesidades que tienen los sujetos de aprendizaje, siendo modificables en cuanto a la perspectiva que se tenga de la pedagogía y los fines que se quieren alcanzar. Para Estébanez (1992) la meta de un modelo pedagógico es "hacer del aprendizaje [...] un elemento que contribuya a la educación de la persona". Ello es relevante pues no siempre que exista un aprendizaje se ha educado. En efecto, la educación es un proceso que va más allá del aprendizaje, tiene una mayor relación con el papel del educando en el entorno que habita:

[...] Un modelo pedagógico hace suyos los principios que promueven la creación de una cultura común en la diversidad, y una situación en la que todas las clases puedan participar en los mismos términos. Se asume que el conocimiento tiene una existencia objetiva, pero se afirma que ha de estar sujeto a una reinterpretación continua mediante un proceso de trabajo personal.

Ahondando sobre el tema de los modelos pedagógicos es posible mencionar que se trata de constructos en los que se establece "un proceso o entidad del cual se supone su existencia aun cuando no sea directamente observable o medible" (Coll, et. al. 1994). En efecto, los modelos son representaciones lo cual supone su existencia sólo en las relaciones que se establezcan entre los actores pedagógicos. Como modelos no son definitivos ni universales, son alternativos y complementarios. Por ejemplo, la teoría de la Modificabilidad Estructural Cognitiva (MEC) y la Experiencia de 
Aprendizaje Mediado (EAM) están cimentadas en varios presupuestos teóricos que tienen su origen el constructivismo, el avance de las ciencias de la cognición y en la teoría sociocultural e histórica. Por lo tanto, un modelo pedagógico es el resultado práctico de las teorías pedagógicas, que dan cuenta al para qué, cuándo y el con qué del acto educativo. Todo modelo pedagógico adopta una postura frente al currículo, en cuanto a sus propósitos, contenidos y secuencias. $Y$ dado que cada una de las teorías es elaborada a partir de un fundamento socio-antropo-psicológico, que da razón del tipo de hombre y de sociedad que se pretende contribuir a formar, toda teoría pedagógica debe poseer también fundamentos teóricos (psicológicos, sociológicos y antropológicos)

Un modelo pedagógico plantea de forma integral un determinado fenómeno; desde el punto de vista teórico-práctico ofrece un marco de referencia para entender implicaciones, alcances, limitaciones y debilidades paradigmáticas que se dan para explicarlo (Gimeno \& Pérez, 1992). En las ciencias sociales los modelos macro y micro intentan describir $y$ entender los fenómenos sociales dados en su estructura, funcionamiento y desarrollo histórico.

De Zubiría (2003) explica que los modelos pedagógicos y su comprensión es importante en la medida que posibilita "reconocer las huellas o rastros que permiten reconstruir aspectos de la vida humana y que sirven de base para la reflexión y la investigación". Un modelo pedagógico es una amplia representación de un fenómeno y a partir de él se pueden abrir espacios para la reflexión dentro del quehacer pedagógico.

Los modelos pedagógicos pueden ser de dos tipos (De Zubiría, 2007): heteroestructurantes y autoestructurantes. Dentro del primer tipo se clasifica el modelo conductista y tradicional, y dentro del segundo, el aprendizaje significativo, el constructivismo o la Experiencia de Aprendizaje Mediado (EAM).

A través del siguiente cuadro se explican las principales características de los modelos pedagógicos heteroestructurantes y autoestructurantes. 
Cuadro 1. Caracteristicas de los modelos heteroestructurantes y autoestructurantes

\begin{tabular}{|c|c|c|}
\hline Elemento & $\begin{array}{c}\text { Concepción en los modelos } \\
\text { heteroesctructurantes }\end{array}$ & $\begin{array}{c}\text { Concepción en los modelos } \\
\text { autoestructurantes }\end{array}$ \\
\hline Educación & $\begin{array}{l}\text { Construcción externa al aula de } \\
\text { clase. }\end{array}$ & $\begin{array}{l}\text { Construcción desde el interior } \\
\text { jalonada desde el propio } \\
\text { estudiante. }\end{array}$ \\
\hline Saber & $\begin{array}{l}\text { Asimilación desde el exterior } \\
\text { basado en la repetición y la copia. } \\
\text { Basado en el docente. }\end{array}$ & $\begin{array}{l}\text { Construcción desde el interior y } \\
\text { jalonada por el estudiante. } \\
\text { Privilegia estrategias por } \\
\text { descubrimiento e invención. } \\
\text { Centrado en la dinámica e interés } \\
\text { de los estudiantes. }\end{array}$ \\
\hline $\begin{array}{l}\text { Sujeto de } \\
\text { aprendizaje }\end{array}$ & Pasivo & Activo \\
\hline $\begin{array}{l}\text { Actuación del } \\
\text { maestro }\end{array}$ & $\begin{array}{l}\text { Espacio para reproducir } \\
\text { conocimiento y favorecer el } \\
\text { trabajo rutinario. }\end{array}$ & $\begin{array}{l}\text { El docente es una guía, un } \\
\text { acompañante. }\end{array}$ \\
\hline $\begin{array}{l}\text { Propósitos y } \\
\text { contenidos }\end{array}$ & $\begin{array}{l}\text { Aprendizaje de informaciones y } \\
\text { normas. }\end{array}$ & $\begin{array}{l}\text { Privilegia la pregunta abierta y la } \\
\text { opinión en la formación } \\
\text { multidisciplinar. }\end{array}$ \\
\hline
\end{tabular}

Fuente: Elaboración propia a partir de De Zubiria (2007)

De la misma forma, De Zubiría (2003) realiza otra clasificación de modelos pedagógicos: el instruccional, el activista y los contemporáneos. El modelo pedagógico instruccional, corresponde a la práctica de la Pedagogía Tradicional. Se identifica por ser la escuela de la obediencia, la puntualidad y el trabajo mecánico como repetitivo, su finalidad consiste en enseñar conocimientos específicos además de las normas aceptadas socialmente. En su Fundamento Psicológico, el niño es considerado como una tabula rasa sobre la que se van imprimiendo desde el exterior saberes específicos.

El modelo pedagógico activista se observa especialmente en la llamada escuela nueva, la cual se caracteriza por la humanización de la enseñanza, al reconocer en el niño sus derechos, capacidades e intereses propios. Otras de sus características son: a) La finalidad de la escuela es preparar para la vida permitiéndole al individuo pensar y actuar a su manera; b) El eje central del proceso es el alumno y sus intereses. Hay autoconstrucción del conocimiento, autoeducación y auto-gobernabilidad. Este modelo carece de una concepción científica de enseñanza; c) En el currículo los programas y los métodos parten de los intereses de los alumnos, cuyos contenidos son la naturaleza y la vida organizados de lo simple y concreto a lo complejo y abstracto; d) El método consiste en la manipulación y el aprender haciendo, ya que el aprendizaje depende la experiencia. No hay diferenciación entre el conocimiento científico y el cotidiano, la acción se generaliza a todas las edades, los recursos permiten la manipulación y la experimentación de tal manera que se invoquen los sentidos y se garantice el aprendizaje y el desarrollo de las capacidades individuales; e) En la relación maestro-alumno existe libertad para hablar y actuar, el maestro no es el ser imponente que lo sabe y lo regula todo.

Los modelos pedagógicos contemporáneos hacen referencia a un conjunto de éstos que se caracterizan por estar basados en las teorías cognitivas. Las aplicaciones pedagógicas de su 
teoría en algunos casos no lograron diferenciarse del activismo, y en otros se han aplicado a áreas particulares. Actualmente, se considera a las corrientes pedagógicas piagetianas como variantes de la escuela nueva y el activismo. El análisis realizado por De Zubiría (2003) concuerda con el de otros autores, donde es posible reconocer a través de la historia tres modelos pedagógicos que se desarrollan de manera notoria:

- Ios modelos disciplinares del siglo XVIII.

- Ios modelos correctivos de comienzos del siglo XX.

- modelos psicológicos con origen a mediados del siglo XX hasta la actualidad.

Becker (2008) aborda tres modelos pedagógicos y epistemológicos para representar las situaciones de enseñanza y aprendizaje. El primero, denominado pedagogía directiva, constituye un modelo de enseñanza-aprendizaje legado por la educación hace más de un siglo, corresponde a las aulas de clase con educandos en silencio y un profesor que tiene la fiel convicción que a través de la palabra, el dictado y la copia se puede transmitir el conocimiento pleno y las habilidades necesarias para afrontar la sociedad del conocimiento de la cual es hoy parte la humanidad. Desde el punto de vista epistémico, el profesor considera al educando como un papel en blanco desconociendo las experiencias y conocimientos previos. En efecto, los hombres son seres socio-culturales marcados por el entorno.

Gráfico 1. Relación maestro-sujeto de aprendizaje en el modelo de la pedagogía directiva

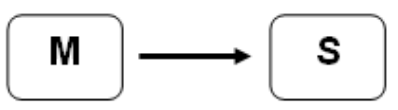

Fuente: Becker (2008)

El segundo modelo, denominado no directiva, supone un papel del profesor restringido a la facilitación del conocimiento, el cual es encontrado por el educando a través de las ayudas y caminos que el profesor diseña para la búsqueda sistematizada. En tal perspectiva de la educación el educando aprende por sí mismo.

Gráfico 2. Relación maestro-sujeto de aprendizaje en el modelo de la pedagogía no directiva

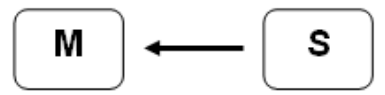

Fuente: Becker (2008)

El tercer modelo, llamado relacional, aborda al profesor como un mediador del conocimiento, quien encuentra en los conocimientos previos de los educandos los cimientos necesarios para la construcción del nuevo conocimiento. Los educandos participan activamente con una acción dinámica del 
profesor. En este modelo hay una fuerte relación entre los sujetos del acto educativo.

Gráfico 3. Relación maestro-sujeto de aprendizaje en el modelo de la pedagogía relacional

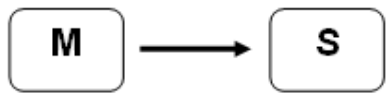

Fuente: Becker (2008)

En los tres modelos de enseñanza-aprendizaje se describen tres perspectivas de la pedagogía. La directiva corresponde al modelo pedagógico tradicional que se basaba en procesos verticales en respuesta a una sociedad basada en la industria y la fábrica, las cuales requerían obreros para laborar largas horas de una forma mecánica y repetitiva. La no directiva es la perspectiva de la pedagogía constructivista en la cual "la organización [de la información y el conocimiento] es siempre el resultado de una interacción necesaria entre la inteligencia consciente y el ambiente" (Von, 1990), en donde el educando logra adaptarse previo el desequilibrio que le produce los conocimientos nuevos. Finalmente, el modelo relacional es equiparable a varios modelos pedagógicos como la Experiencia de Aprendizaje Mediado (EAM) de Feuerstein (1991) o la teoría socio-cultural e histórica de Vygotsky (1995).

El hecho que los modelos pedagógicos se puedan analizar de esta manera sugiere que los mismos no pueden comprenderse fuera de un tiempo-espacio determinado donde nacen y se desarrollan cada uno (Varela, 1992). Clasificándolos dentro del modelo de Zubiría (2007) se puede afirmar que los dos primeros caben dentro del modelo heteroestructurante, y los últimos dentro del modelo autoestructurante.

El enfoque pedagógico conductista fue desplazado por el modelo pedagógico propuesto por Piaget (2005). Para Piaget, citado por Weissmann (2007), la inteligencia es un "proceso activo, organizado y ordenado" en el cual se realizan dos actividades para llegar al conocimiento del objeto: asimilación y acomodación (Avendaño \& Parada, 2011), los cuales conducen a la adaptación del sujeto al medio. A medida que avanza la edad del sujeto, éste puede trabajar con información más compleja y abstracta. Para Piaget (2005) el lenguaje es esencial en la construcción del conocimiento en el sujeto.

El lenguaje también fue objeto de estudio para Vygostki (1985) quien reveló la importancia o relevancia del lenguaje en los procesos de aprendizaje. Por esta razón, las deficiencias en el ámbito del lenguaje limitan los procesos de pensamiento de las personas. En efecto, el lenguaje ha permitido al hombre su construcción como ser socio-cultural. Vygostki (1985) en su teoría desarrolló el concepto de Zona de Desarrollo Próximo (ZDP), la cual entiende como el potencial del niño(a) para resolver un problema de forma independiente y la capacidad para hacerlo mediante la guía de un adulto. La ZDP es el potencial del sujeto de aprendizaje para adquirir determinados 
conocimientos y hacer uso de ellos en un contexto específico. Esta contribución de Vygostki (1985) tiene relación con los postulados de Feuerstein (1963) en lo que se refiere a la Experiencia de Aprendizaje Mediado (EAM). Con ello se quiere resaltar que la concepción de Feuerstein sobre el desarrollo humano, la educación y la pedagogía son relevantes y tienen un alto grado de validez en la actualidad para múltiples escenarios de la formación, entre ellos, el de la educación ambiental, pues como advierte Orellana (2002) éstos ofrecen un "marco epistemológico para la comunidad de aprendizaje en educación ambiental".

\section{LAS CORRIENTES DE LA EDUCACION AMBIENTAL}

La Conferencia Intergubernamental sobre Educación Ambiental en Tbilisi (URSS) de 1977 estableció que ésta debía adoptar una perspectiva holística que incluyera la dimensión sociocultural, ecológica, política, económica y demás, a fin de propiciar la solución de problemas específicos. Otros instrumentos internacionales con posterioridad también advirtieron la problemática como la Declaración de Río de 1992. La directriz ofrecida por la Conferencia de 1977 va enfocada a la integración disciplinar, a lo que Morin (1999) llamaría la complejidad del conocimiento. La misma Conferencia invita al cambio de los enfoques pedagógicos anclados en la enseñanza por enfoques basados en la acción y en los problemas. Además de ello, sugiere que los contenidos, métodos y materiales específicos se adapten a las necesidades de los educandos.

En este contexto de normas internacionales ratificadas por los Estados se han elaborado múltiples representaciones alrededor de la educación ambiental. Ya desde los años 40's se inició la construcción de corrientes en la educación ambiental con un auge en los 70's y 80’s cuando se hacía más evidente la problemática del medio ambiente (Orellana \& Fauteux, 2002). Como ejemplo de ello se encuentra la Conferencia en Tbilisi en 1977.

Dentro de las corrientes para la educación ambiental más destacadas se encuentran la naturalista, conservacionista, resolutiva, sistémica, científica, humanista y moral-ética (Sauvé, 2004). 
Cuadro 2. Corrientes de la educación ambiental

\begin{tabular}{|l|l|}
\hline \multicolumn{1}{|c|}{ Corriente } & \multicolumn{1}{c|}{ Conceptualización } \\
\hline Naturalista & $\begin{array}{l}\text { Esta corriente se desarrolla en un enfoque del hombre con la } \\
\text { naturaleza desde las dimensiones cognitiva, experimental, afectiva } \\
\text { y artística. Para la corriente naturalista hay un valor en la naturaleza } \\
\text { más allá de sus recursos. Además, la naturaleza es un medio para } \\
\text { la formación de los sujetos de aprendizaje. }\end{array}$ \\
\hline Conservacionista & $\begin{array}{l}\text { La corriente promociona la conservación de los recursos sobre todo } \\
\text { en espacios donde éstos son escasos. Se desarrolla a través de } \\
\text { proyectos que gestionan acciones para la generación de } \\
\text { comportamientos que contribuyan a la equidad social. }\end{array}$ \\
\hline Resolutiva & $\begin{array}{l}\text { La corriente asume la educación ambiental como un conjunto de } \\
\text { problemas, los cuales debe asumir la sociedad de manera definitiva, } \\
\text { ya que, las dificultades medio ambientales son apremiantes. }\end{array}$ \\
\hline Sistémica & $\begin{array}{l}\text { La corriente reconoce el ambiente como un sistema complejo desde } \\
\text { un énfasis ecológico. Para un cambio desde la educación es } \\
\text { necesario acceder al sistema ambiental e identificar y analizar las } \\
\text { vías de evolución y de ruptura. }\end{array}$ \\
\hline Humanista & $\begin{array}{l}\text { Esta corriente asume la educación ambiental desde el método } \\
\text { científico, para lo cual busca observar los problemas de la } \\
\text { naturaleza y establecer relaciones de causa-efecto. }\end{array}$ \\
\hline Moral-ética & $\begin{array}{l}\text { Enfatiza en la perspectiva humana de la educación ambiental } \\
\text { traducida en la relación de la naturaleza con la cultura en las } \\
\text { dimensiones económico-políticas e históricas. }\end{array}$ \\
\hline $\begin{array}{l}\text { Considerada el fundamento de las relaciones entre el hombre y la } \\
\text { naturaleza; es desarrollada a través de los estudios de caso sin } \\
\text { desconocer el valor de la formación social y científica. }\end{array}$ \\
\hline
\end{tabular}

Fuente: Elaboración propia a partir de Sauvé (2004)

Las corrientes elaboradas en torno a la educación ambiental asumen el medio ambiente desde diversas perspectivas al igual que la concepción de hombre. Las relaciones entre el uno y otro evidencian una posición del ser humano frente al medio ambiente. Por ejemplo, la corriente moral-ética asume al hombre como un sujeto ético y moral, cuyo discernimiento y voluntad puede crear comportamientos amigables con el medio ambiente; mientras que la resolutiva considera al hombre un ser capaz de crear instrumentos y desarrollar acciones para solucionar definitivamente los graves problemas que ha ocasionado al medio ambiente. Sauvé (2001) hace una tipología de las representaciones del medio ambiente el cual puede ser observado, analizado y comprendido como naturaleza, recurso, problema, medio de vida, contexto de significación, sistema, territorio, paisaje, biosfera y proyecto comunitario. 


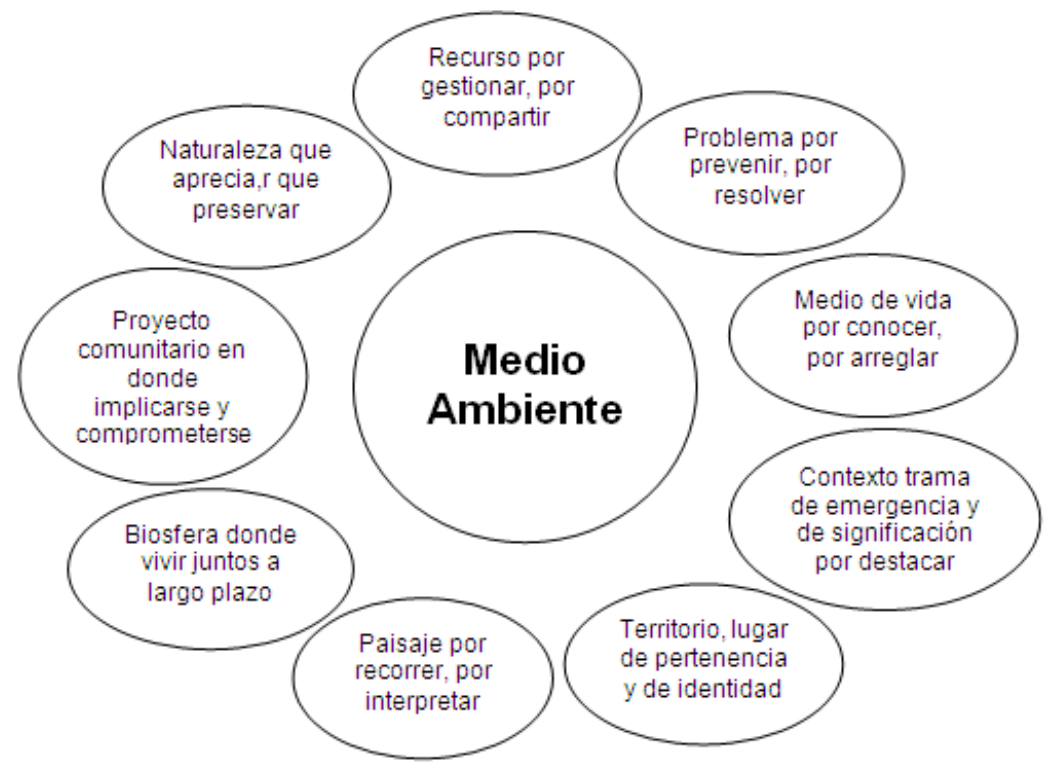

Fuente: Sauvé (2001)

Con la conferencia de Tbilisi se abordó la preocupación por los graves daños de las acciones del hombre sobre el medio ambiente, en ella se genera la concepción de la educación ambiental como instrumento para brindar conocimientos y corregir comportamientos del hombre hacia la naturaleza. La educación en general, en especial la educación ambiental, es vista en la actualidad como una potente herramienta para la solución de muchos problemas medioambientales y para la reconstrucción de una humanidad que respete las condiciones y el normal funcionamiento de la naturaleza, convirtiéndose en una estrategia para alcanzar el desarrollo sostenible (Vega \& Álvarez, 2005), tema muy debatido en la actualidad.

Algunas consideraciones que se han debido tener en consideración por la educación ambiental, aparte del cambio de perspectiva sobre el hombre y el medio ambiente, son los avances en los campos de la tecnología, la técnica y la educación en general. Los aportes desde la ciencia de la psicología así como los descubrimientos sobre la cognición, son elementos de necesaria revisión para la modificación de los modelos pedagógicos. El desplazamiento de la pedagogía tradicional y conductista posibilita un cambio de actitud del maestro y la visión de lo educativo hacia nuevos horizontes: "la formación de profesores, animadores y otros dinamizadores en educación ambiental es fundamental para el desarrollo de este campo y responde a una triple problemática: ambiental, social y educativa" (Sauvé, 2003). Desde la perspectiva de Sauvé el modelo la Experiencia de Aprendizaje Mediado de la Teoría de la Modificabilidad Estructural Cognitiva puede ser en el camino para la consolidación de los objetivos de la educación ambiental 


\section{LA TEORÍA DE LA MODIFICABILIDAD ESTRUCTURAL COGNITIVA (MEC) Y LA EXPERIENCIA DE APRENDIZAJE MEDIADO (EAM)}

La Teoría de la Modificabilidad Estructural Cognitiva es el resultado de los estudios realizados desde hace más de 50 años por Reueven Feuerstein, precisamente en los años posteriores a la II Guerra Mundial cuando las consecuencias graves y devastadoras afectaron a la población en todas sus dimensiones: social, cultural, emocional, psicológica intelectual (Orrú, 2003). Para Noguez (2002) en entrevista con Feuerstein la Modificabilidad Estructural Cognitiva

[...] creció a partir de mi interés por ver cómo la gente con bajo rendimiento, y en ciertos casos extremadamente bajo, llega a ser capaz de modificarse mediante procesos cognoscitivos para adaptarse a las exigencias de la sociedad. El trabajo con esta gente me ha demostrado que la modificabilidad cognitiva es de hecho posible, así que intenté buscar la base teórica para respaldar nuestros datos empíricos. La teoría de la MCE ha evolucionado con los años y ha permitido el desarrollo de una variedad de aparatos cognoscitivos que sirven como los pilares de la teoría.

Para Feuerstein (1963) "el organismo humano es un sistema abierto que en su evolución adquirió la propensividad para modificarse a sí mismo, siempre y cuando exista un acto humano mediador", esto significa que a pesar de las dificultades que tenga el sujeto para el aprendizaje, existe la posibilidad de ser mediador de tal manera que aquellos factores que afectan de forma negativa su aprendizaje puedan ser superados. En resumen, se trata de una teoría integradora e inclusiva en la que sólo basta una acción del maestro acorde a las necesidades de la población en el marco de unos criterios que ofrece la teoría en mención. Por los importantes avances en las últimas décadas, la teoría de Feuerstein ha tenido una aceptación bastante amplia entre los pedagogos, toda vez que sus postulados adquieren mayor validez a partir de los resultados de las investigaciones que se realizan acerca de la naturaleza y funcionamiento del cerebro. Al respecto menciona Ramírez y Roa (2003):

[...] el ser humano se caracteriza por su alto nivel de flexibilidad. Las teorías que están apareciendo sobre el funcionamiento del sistema nervioso y de la fisiología del cerebro así lo están confirmando, cuando nos hablan de plasticidad y flexibilidad del ser humano. Según esto, el individuo tiene el potencial oculto para aprender y puede modificarse a sí mismo si lo desea $[\ldots]$ 
En esta misma línea Feuerstein señala la relación entre inteligencia y modificabilidad:

[...] la inteligencia (es) un proceso en lugar de una entidad fija, inmutable y concreta. Proceso lo bastante amplio como para abarcar una enorme variedad de fenómenos que tienen en común la dinámica y la mecánica de la adaptación, en su acepción más genérica; es decir los cambios que sufre el organismo como respuesta a la aparición de una nueva situación que requiere tales cambios. Esta adaptabilidad del organismo es a la que nos referiremos por modificabilidad. Esta propensión al cambio, esta flexibilidad y plasticidad, es la que entenderemos como inteligencia.

La Experiencia de Aprendizaje Mediado (EAM) de la teoría de la Modificabilidad Estructural Cognitiva (MEC) propone un modelo que inspira la esperanza de aquellos que han sido excluidos por la deprivación cultural, es decir, por la incapacidad del sujeto para responder adecuadamente a los estímulos (Pilonieta, 2010) y que tienen su causa en diversos factores. Para Feuerstein y Rand (1974) dichos factores pueden ser de dos clases: etiológicos distales y etiológicos próximos (Gráfico 5). Los primeros relacionados con los aspectos orgánicos, biológicos y socioculturales del sujeto. Los segundos corresponden al acto pedagógico de los maestros que en muchas oportunidades más que educar puede obstaculizar y disminuir la capacidad de aprendizaje y modificación de la persona. Los factores etiológicos son determinantes del desarrollo cognitivo diferencial, en otras palabras, son determinantes para que el organismo tenga "formas bajas y niveles [...] inadecuados de funcionamiento cognitivo hasta niveles superiores de pensamiento" (Prieto, 1989). 
Gráfico 5: Factores etiológicos asociados a la Mediación

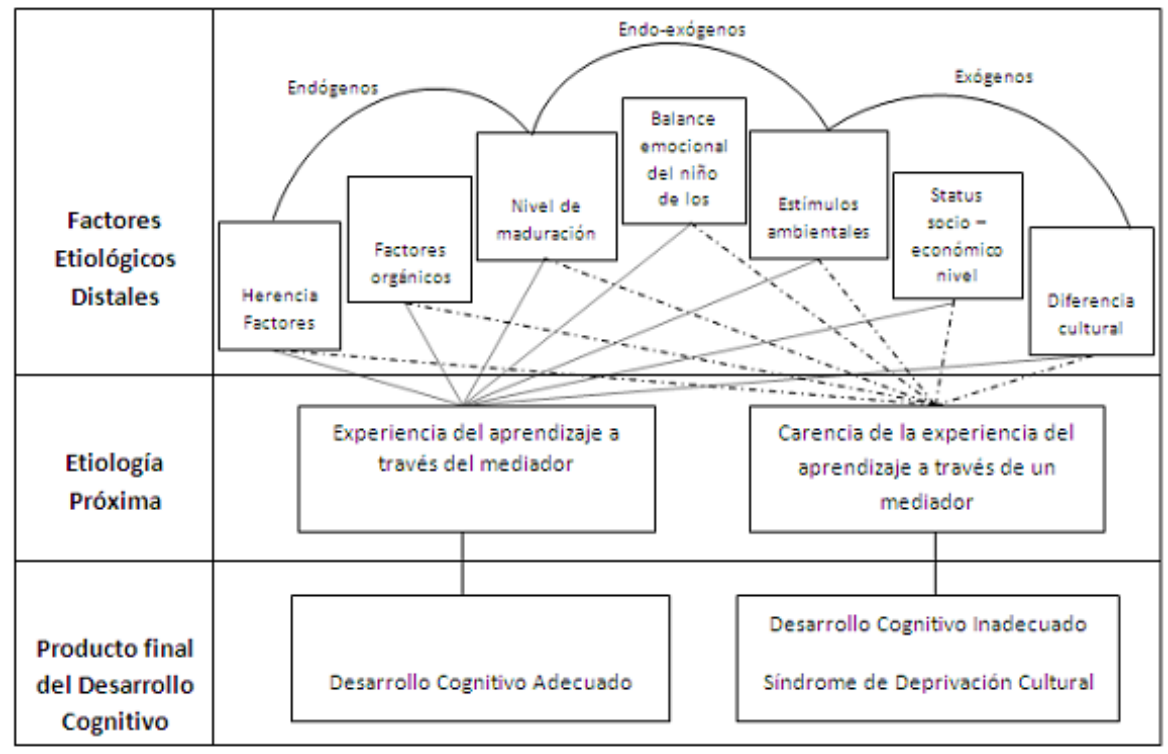

Fuente: Feuerstein y Rand, 1974

La teoría de la MEC, según Varela, Gramacho y Melo (2006) defiende tres grandes ideas: la doble ontogenia del ser humano: biológica y socio-cultural; el comportamiento humano como un estado; y, finalmente, la concepción neurocientífica del cerebro humano como un ente flexible y dinámico. Si se analizan estos aportes de los autores se puede inferir que como teoría aplicada a la educación y a la educación ambiental es de gran impacto para la construcción de conocimientos desde una perspectiva socio-cultural y cognitiva. Esto sólo es posible con la Experiencia de Aprendizaje Mediado (EAM), el cual es el dispositivo para la verdadera modificabilidad del sujeto.

De acuerdo a Noguez (2002) la Experiencia de Aprendizaje Mediado no es otra cosa que la "calidad de la interacción del ser humano con su ambiente. La EAM [...]"conlleva a la explicación de los procesos cognoscitivos como subproducto de la transmisión cultural" (Noguez, 2002). Lo que aquí se observa es una de las modalidades en que los sujetos pueden aprender, un modelo de aprendizaje dinámico y flexible del cual hablara Becker (2008) ya abordado con anterioridad. 
Gráfico 6. Modelos de aprendizaje

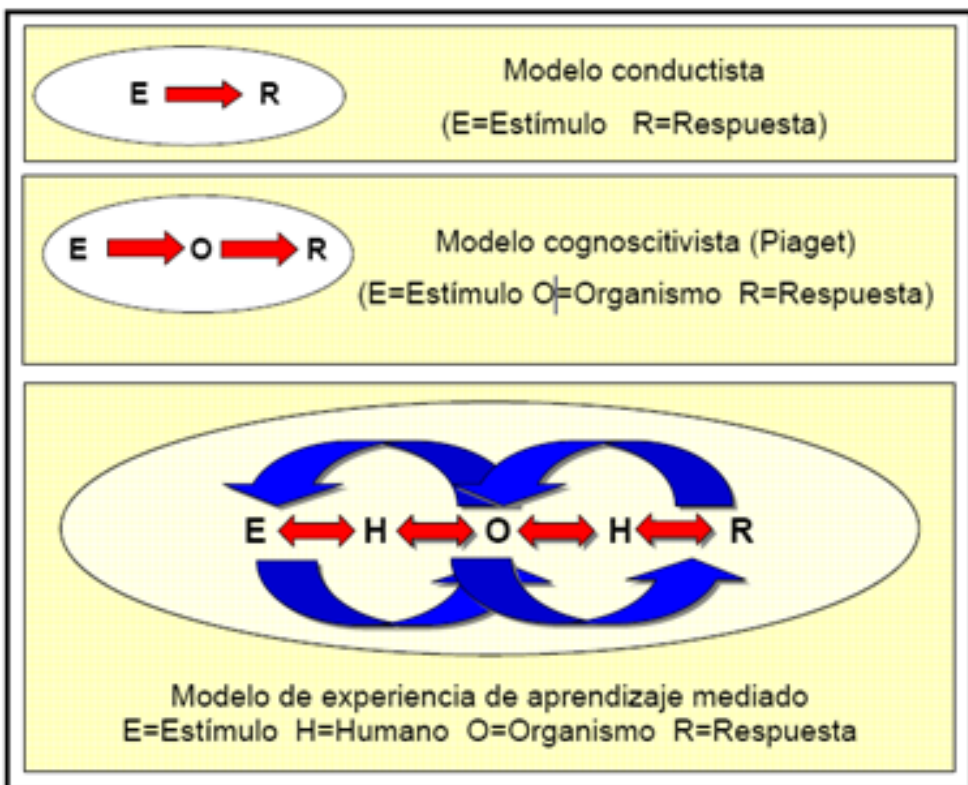

Fuente: Noguez, 2002

La gráfica explica que el aprendizaje se produce cuando hay una exposición de estímulos provenientes del medio sobre el sujeto. En el modelo conductista y tradicional el estímulo era directo sin intervención de agentes mediadores. En el modelo cognoscitivo de Piaget existe una acción del sujeto entre el estímulo y el sujeto de aprendizaje, pero no hay retroalimentación, siendo de corte vertical. En el último, propuesto por Feuerstein hay una interacción del maestro entre los estímulos y el sujeto, en donde analiza su respuesta, reflexiona en torno al sujeto y actúa en función de mejorar la calidad del estímulo. En efecto, como explica Ramírez y Roa (2003)

El ser humano, desde que nace, interactúa con su medio de dos formas distintas: por un lado, el acercamiento a su entorno puede realizarse autónomamente, sin intermediarios. En este caso, el sujeto se expone directamente a los estímulos y elabora una respuesta en función de éstos; por otro lado, la persona humana puede encontrarse con un adulto (padre, madre, maestro, etc.) que actúe como mediador entre el organismo y el entorno.

Los aportes de Vygostky (1985) así como los de Piaget (2005) han servido para alimentar la teoría de Feuerstein. Del primero ha tomado el carácter socio-cultural del sujeto y la influencia del medio sobre el mismo, y del segundo los procesos internos desde la perspectiva cognitiva. De igual manera con los dos formula la modificabilidad en términos de adaptación del hombre. 
La Experiencia de Aprendizaje Mediado constituye por lo tanto una concepción teórica que explica el vínculo y la interacción humana en la construcción de nuevos estados del sujeto, su objetivo es generar efectos sobre organismos para modificar el pensamiento de acuerdo al criterio de auto-plasticidad ${ }^{2}$. La ausencia de este tipo de procesos tiene efectos negativos sobre las personas, quienes adquieren rigidez en sus procesos mentales.

Para que la Experiencia de Aprendizaje Mediado (EAM) tenga un impacto positivo sobre el sujeto intervenido, se debe atender a varios criterios. Afirma Feuerstein (1994), "por lo menos tres características importantes deben caracterizar la interacción: la intencionalidad y la reciprocidad, la mediación del significado, y la trascendencia".

- Intencionalidad y reciprocidad (Intentionality and Reciprocity): Es la claridad del mediador sobre los objetivos a alcanzar y deben ser compartidos con el sujeto involucrado en dicho proceso. Así, la intencionalidad no puede estar ligada sólo a la transmisión de contenidos, por el contrario, se debe dirigir hacia la construcción de nuevos estados en la persona y en la eliminación de la deprivación cultural. Por lo tanto, la intención está contextualizada en la estructuración o modificación a nivel cognitivo.

- Mediación de la trascendencia (Mediation of Meaning): Es la posibilidad del sujeto de aplicar, en nuevos contextos y situaciones, aquello que ha interiorizado y ha hecho parte de su desarrollo como producto del proceso de formación.

- Mediación significado (Mediation of Meaning): Es manejar el sentido de la actividad, su importancia, posibles aplicaciones y el interés del sujeto mediado sobre el mismo proceso. Para Mentis (1997) "la mediación del significado está relacionada con imprimir valor y energía a la actividad u objeto, volviéndose relevante para el mediado".

Estos criterios constituyen el marco de un modelo pedagógico que tome como dimensiones sociocultural y cognitiva del sujeto. Es una acción sobre el sujeto de una manera dinámica que permite, aun, desde la educación ambiental traer resultados positivos.

\section{EL MODELO PEDAGÓGICO PARA LA EDUCACIÓN AMBIENTAL}

El fin e intención educativa en lo ambiental es el reconocimiento del medio ambiente como un todo del cual hace parte el ser humano, éste que puede mejorar su actuación y actitud frente a la solución de los problemas que ya son latentes y la preservación de aquello que aún se conservan. Según el Ministerio de Ambiente y el Ministerio de Educación Nacional (2002) con tales procesos formativos se busca que el 
Individuo comprenda las relaciones de interdependencia con su entorno, a partir del conocimiento reflexivo y crítico de su realidad biofísica, social, política, económica y cultural para que, a partir de la apropiación de la realidad concreta, se puedan generar en él y en su comunidad actitudes de valoración y respeto por el ambiente.

\section{Gráfico 7. Intencionalidad del modelo pedagógico}

\section{Fin-Intención: Relación cercana y amigable del hombre con el medio ambiente. \\ Redescubrir el significado del mismo y su papel como actor político.}

Fuente: autor

El fin o intención propuesta conduce a identificar las dimensiones de desarrollo del sujeto. Así, es fundamental un trabajo integral desde múltiples dimensiones: emocional, afectivo, cognitivo, socio-cultural.

Gráfico 8. Dimensiones a desarrollar con el modelo pedagógico

Fin-Intención: Relación cercana y amigable del hombre con el medio ambiente.

Redescubrir el significado del mismo y su papel como actor político.

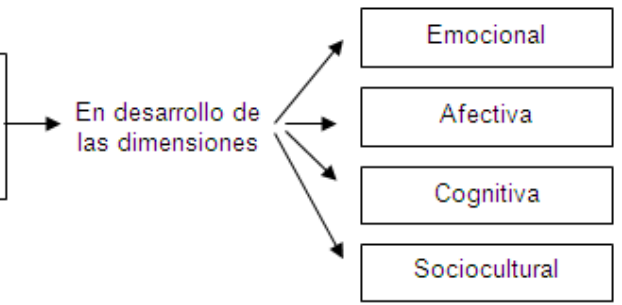

Fuente: autor

Las dimensiones de desarrollo con la educación ambiental se realizan a través de los contenidos, los cuales se consideran medios. El aprendizaje no es sinónimo de educación y es precisamente la concepción contraria la que se ha mantenido haciendo de la educación un proceso en el que se busca la transcripción de textos y la memorización de los mismos, creyendo ingenuamente, que se ha cumplido con la función de educar. En el modelo pedagógico propuesto los contenidos medio-ambientales son medios que sirven de enlace con la construcción de una nueva identidad del sujeto que permite armonizar las relaciones del mismo con el medio ambiente y pueden ser de tres tipos: conceptuales, actitudinales y procedimentales. 


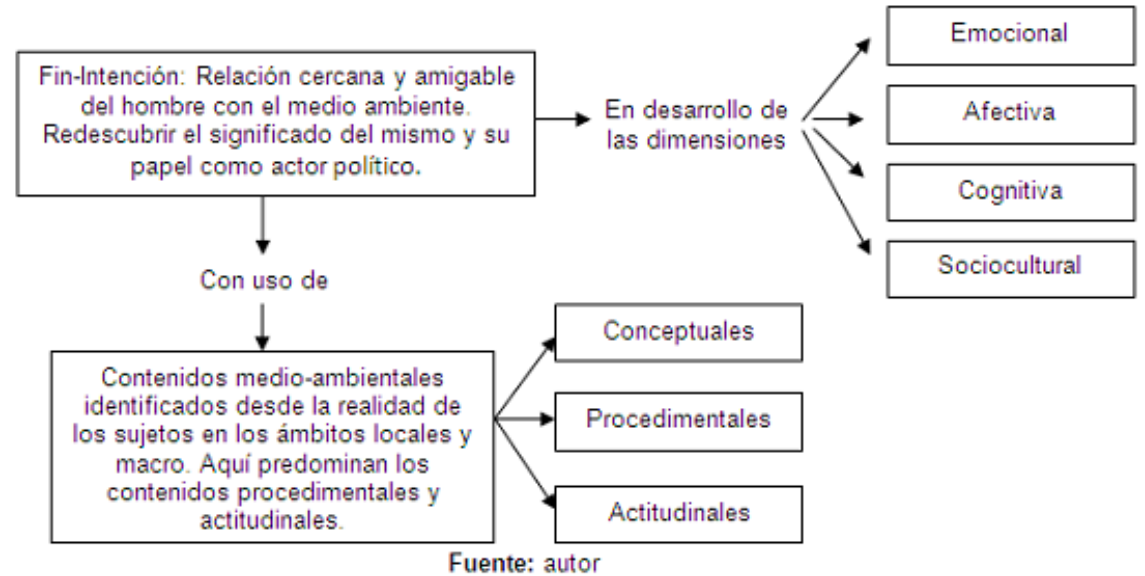

En cuanto a la secuencia de los contenidos estos deben atender al grado de maduración de los niños, niñas y jóvenes, así como a los aspectos etiológicos distales. Por lo tanto, la secuencia tendrá como criterios la complejidad y la abstracción. Complejidad en términos de la calidad y cantidad de la información, y abstracción considerando lo concreto de los objetos de estudio a lo más abstracto.

Gráfico 10. Criterios para los contenidos del modelo pedagógico

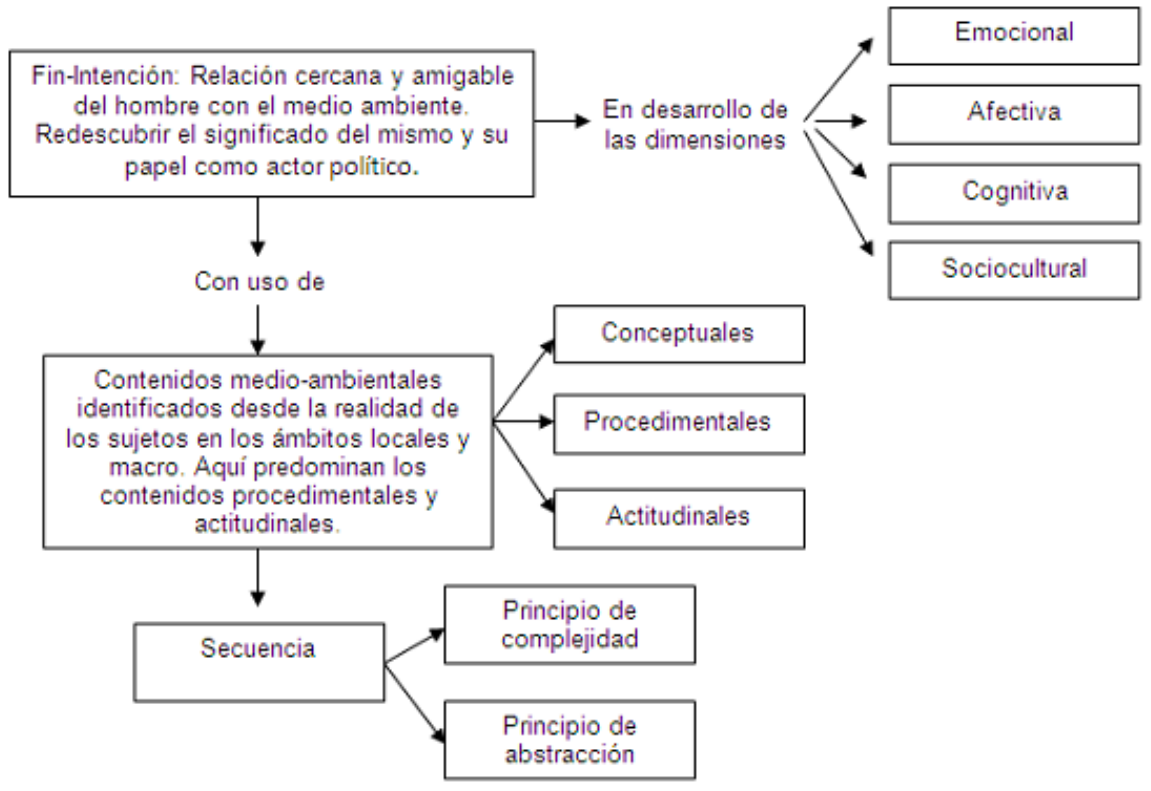

Fuente: autor

La metodología a implementar, así como las estrategias pedagógicas y la pedagogía, están nutridas por la Experiencia de Aprendizaje Mediado bajo el principio que todo organismo es modificable siempre y cuando exista una acción mediadora sobre el sujeto. Finalmente, la evaluación como elemento esencial del modelo pedagógico se atiende como un proceso permanente que elimina la calificación como medio de exclusión y se percibe como un instrumento del cual hace uso el 
educando para comprender su evolución y generar verdaderas experiencias de metacognición. Desde esta perspectiva la evaluación es guiada por el maestro pero ejercida autónomamente por el educando.

Gráfico 11. Modelo pedagógico para la educación ambiental

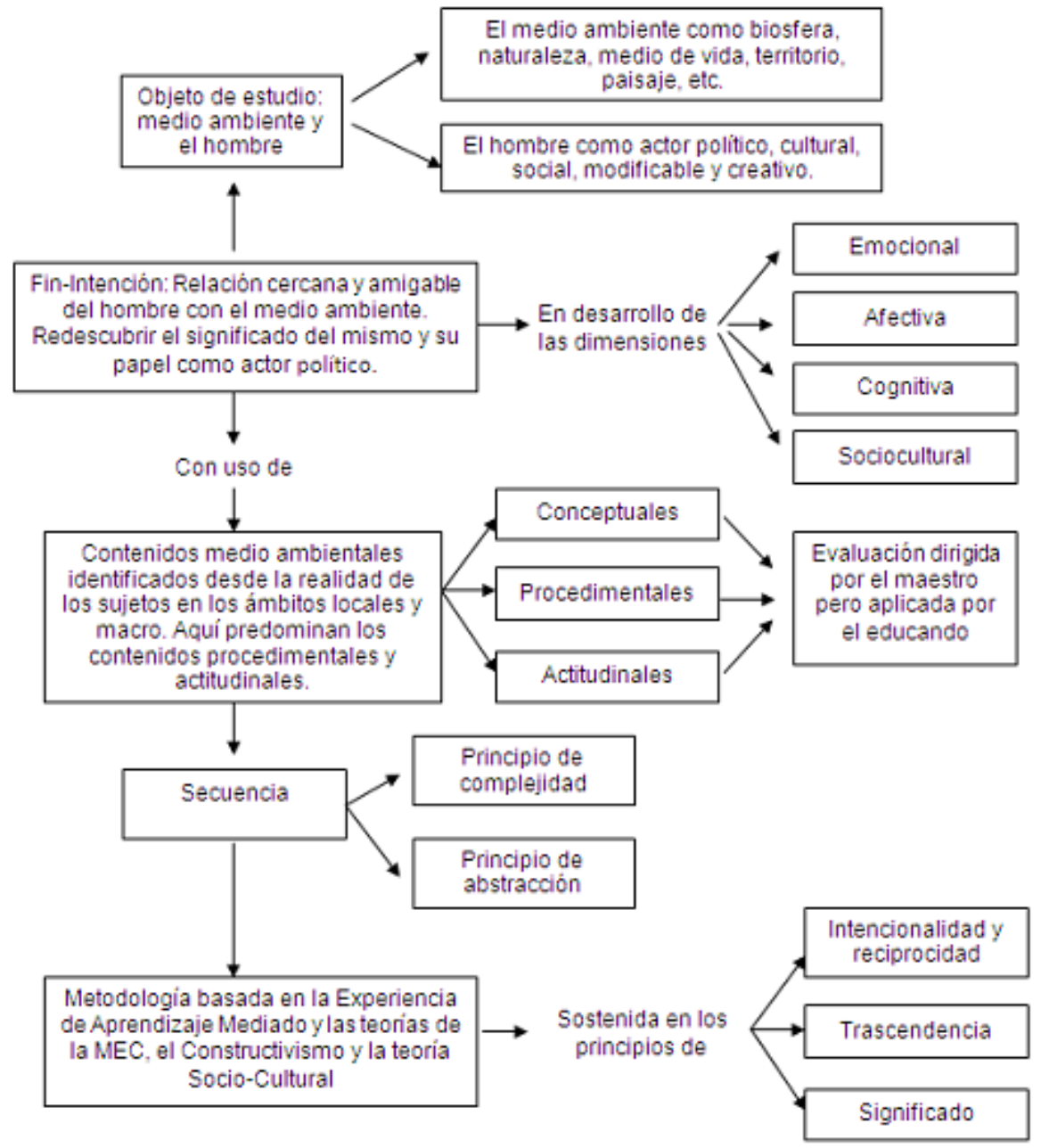

Fuente: autor

\section{CONCLUSIONES}

La educación del siglo pasado ha variado notablemente y con ello los modelos educativos y pedagógicos. Estos elementos novedosos que traen consigo los avances de las ciencias que apoyan la educación permite la construcción de nuevas y mejores formas para concretar la formación de los sujetos. De esta manera, la educación ambiental puede formularse en los mismos términos desde las diversas perspectivas que ofrecen las múltiples corrientes que sobre la educación ambiental se han creado.

Se ha ideado un modelo pedagógico atendiendo a los principales aportes teóricos en pedagogía como los formulados 
por Feuerstein en colaboración del modelo constructivista y sociocultural. En el modelo se deja plasmado el papel de los actores educativos desde un enfoque político y cultural, esencial para una formación idónea. Con los principios y criterios allí planteados, los múltiples problemas medioambientales de los que es parte hoy la humanidad pueden ser superados a mediano y corto plazo.

\section{BIBLIOGRAFÍA}

- Avendaño-Castro, W. R. \& Parada-Trujillo, A. E. (2011). Un modelo pedagógico para la reproducción y transformación cultural en las sociedades del conocimiento. En: Investigación y Desarrollo, Vol. 19, No 2 ISSN 0121-3261. pp. 398-413.

- Avendaño-Castro, W. R. (2011). Responsabilidad Social Corporativa (RSC) y desarrollo sostenible: una mirada desde la Declaración de Río de 1992. Respuestas, Vol. 16 (2). pp. 45-59.

- Becker, Fernando (2008); Modelos pedagógicos e modelos epistemológicos. pp. 45-56. En: Karkotli, Gilson (Comp.); Metodologia. Construção de uma Proposta Científica. Curitiba: Camões. p. 80

- Capra, F. (2002). Las conexiones ocultas: implicaciones sociales, medioambientales, económicas y biológicas de una nueva visión del mundo. Barcelona: Anagrama

- Coll, C.; Pozo, J. I.; Sarabia, B. y Valls E. (1994). Los contenidos en la Reforma. Enseñanza y aprendizaje de conceptos, procedimientos y actitudes. Madrid: Santillana. p. 202

- Conferencia Intergubernamental sobre Educación Ambiental en Tbilisi (URSS). (1977).

- Declaración de Río sobre medio ambiente y desarrollo sostenible, 3 al 14 de junio 1992.

- De Zubiria J. (2003). Modelos Educativos Pedagógicos y Didácticos (Vol. II). Bogotá: Ediciones SEM.

- De Zubiría, J. (2007). Tratado de pedagogía conceptual: Los modelos pedagógicos. Bogotá: Fondo de Publicaciones Bernardo Herrera Meriño. p. 160

- Durkheim, E. (1976). La educación como socialización. Salamanca: Sígueme. 274 p.

- Dickson, D. (1978). Tecnología alternativa y políticas del cambio tecnológico. Madrid: Blume.

- Estébanez-Estébanez, C. (1992). Un modelo pedagógico en la enseñanza del inglés. En: revista Universitaria de Formación del Profesorado, No 15. pp. 173-181.

- Feuerstein, R. (1963). Children of the Melah. Sociocultural deprivation and its educational significance. Jerusalem: Szold Foundation.

- Feuerstein, R. (1980). Instrumental Enrichment: An intervention program for cognitive modifiability, Ilinois: Scott Foresman and Company.

- Feuerstein, R. (1991). Mediated Learning Experience (MLE): theoretical, psychosocial and learning 
implications. Londres: Freund Publishing House Ltd. p.390

- Feuerstein, R. (1994). Mediated Learning Experience (MLE): theoretical, psychosocial and learning implications. Second edition, Freund Publishing House Ltd.

- Feuerstein, R. \& Rand, Y. (1974); Mediated Learning Experiences: An out-line of the proximal etiology for differential development of cognitive functions. International Understanding, L. Gold Fein (ed).

- Flórez, Rafael (1999). Evaluación Pedagógica y Cognición. Bogotá: McGraw-Hill. p. 226

- Forero, F-. (2001); La Profesionalización del maestro y la evaluación de sus competencias. Bogotá: Universidad Pedagógica Nacional.

- Foucault, Michel (1994). Dialogue sur le pouvoir (pp. 464477). En: Foucault, Michel. Dits et écrits. Vol. III. Paris: Gallimard.

- Gimeno-Sacristán J. \& Pérez-Gómez, Á. (1992). Comprender y transformar la enseñanza. Madrid: Morata. p. 448

- Hinojosa-Navarro, R.; López-Martínez, A. \& BarrosoFlores, P. (1998); El análisis de incidentes críticos en la formación inicial de maestros. En: Revista electrónica interuniversitaria de formación del profesorado, Vol. 1, No 1.

- Kanitscheider, B. (2001). El papel integrador de la autoorganización. En: Argumentos de Razón Técnica, No. 4. pp. 117-134.

- Mentis, M. (Coord.) (1997). Aprendizagem mediadu dentro e fora da sala de aula. Programa de pesguisa eognitiva, Divisdo de educaçdo especials'zada da Universidadt de Witwatersand, África do Sul. Sáo Paulo: Instituto Pieron de Psicologla Aplicada.

- Ministerio de Ambiente y Ministerio de Educación Nacional (Colombia) (2002). Politoca Nacional de Educación Ambiental SINA. Bogotá: Ministerio de Educación. p. 69

- Ministerio de Educación-Gobierno de la ciudad de Buenos Aires (2009); Educación ambiental: de la conservación a la formación para la ciudadanía. Buenos Aires: Ministerio de Educación.

- Morin, Edgar (1999). Los siete saberes necesarios para la educación del futuro. París: UNESCO. p.68

- Nasr-Seyyed H. (1982). Hombre y naturaleza. La crisis espiritual del hombre moderno. Buenos Aires: Kier. p.145

- Noguez-Casados, S. (2002). El desarrollo potencial de aprendizaje. Entrevista a Reuven Feuerstein. En: Revista Electrónica de Investigación Educativa, 4(2). Consultado el 7 de marzo del 2011. Desde: http://redie.uabc.mx/vol4no2/contenidonoguez.html.

- Orellana-Isabel (2002). La estrategia pedagógica de la comunidad de aprendizaje, definiendo sus fundamentos, sus prácticas y su pertinencia en 
educación ambiental En: Sauvé, I.; Orellana, I.; Sato, M. (2002). Textos escolhidos em educação ambiental de uma América à outra. Montreal: Les Publications EREUQAM. pp. 221-231.

- Orellana, I. \& Fauteux, E. (2002); La educación ambiental a través de los grandes momentos de su historia En: Sauvé, I.; Orellana, I.; Sato, M. Textos escolhidos em educação ambiental de uma América à outra. Montreal: Les Publications ERE-UQAM. pp. 39-62.

- Organización de las Naciones Unidas para la Educación, la Ciencia y la Cultura (UNESCO) (1998). Hacia una educación sin exclusiones. Santiago de Chile: Oficina Regional de Educación de la UNESCO.

- Osset, Rodrigo (2009). El secreto de Dios, vida del hombre y del cosmos. Madrid: Visión Libros. p. 364

- Organización de las Naciones Unidas para la Educación, la Ciencia y la Cultura (UNESCO) y Programa de Naciones Unidas para el Medio Ambiente (PNUMA) (1977). Conferencia Intergubernamental sobre Educación relativa al Medio Ambiente. Tbilisi (URSS), 14-26 de octubre.

- Orrú, S. E. (2003). Reuven Feuerstein y la teoría de la Modificabilidad Cognitiva Estructural. En: Revista de Educación, No. 332. pp. 33-54. Consultado el 9 de marzo del 2012.2 Desde: http://www.doredin.mec.es/documentos/0082004300 72.pdf

- Piaget, J. (2005). Inteligencia y afectividad. Buenos Aires: Aique

- Pilonieta, P. G. (2003). La teoría de la Modificabilidad Estructural Cognitiva y su influencia en la pedagogía para el desarrollo de las inteligencias y las comunidades de aprendizaje. En: Asociación Colombiana para el Avance de las Ciencias del Comportamiento (ABA Colombia). Consultado el 20 de abril del 2011. en: www.abacolombia.org.com

- Pilonieta P. G. (2010); Modificabilidad Estructural Cognitiva y educación. Bogotá: Magisterio. p. 274

- Porto-Gonçalves, C. W. (2000). Las múltiples y contradictorias practicas de la educación ambiental. En: Formación ambiental, Vol. 12 (26).

- Sauvé, L. (2004). Una cartografía de corrientes en educación ambiental. En: Sato, M. \& Carvalho, I. (Eds) (2004). A pesquisa emeducação ambiental: cartografias de umaidentidade narrativa emformação. Porto Alegre: Artmed.

- Sauvé, L. (2003); Perspectivas curriculares para la formación de formadores en educación ambiental. Ponencia presentada en el I Foro Nacional sobre la Incorporación de la Perspectiva Ambiental en la Formación Técnica y Profesional, Universidad Autónoma de San Luis de Potosí (México) del 9 al 13 de Junio.

- Ramírez-Fernández, S. \& Roa-Venegas, J. Ma (2003). El Programa de Enriquecimiento Instrumental de 
Feuerstein: Una aproximación teórica. En: Revista Euphoros, Número 6.

- Thompson, J. B. (1998); La Media y la Modernidad. Una Teoría de los Medios de Comunicación. Buenos Aires: Paidós.

- Varela, A.; Gramacho, A.; Melo, C. (2006). Programa de Enriquecimiento Instrumental (PEI): alternativa pedagógica que responde al desafío de calidad en educación. Revista Diversitas -Perspectivas en psicología- vol. 2 (2).

- Varela, J. (1992). Categorías espacio-temporales y socialización escolar: del individualismo al narcisismo. En: revista de Educación. Número 298: pp. 7-29.

- Vazquez \& Bárcena (2000). Pedagogía Cognitiva la educación y el estudio de la mente en la Sociedad de la Información. En: Teoría de la Educación: Educación y Cultura en la Sociedad de la Información, Número. 1. Consultado el 9 de marzo del 2012. Desde: http://campus.usal.es/ teoriaeducacion/rev_numero 01/articulo6.html.

- Vega, P. \& Álvarez, P. (2005). Planteamiento de un marco teórico de la Educación Ambiental para un desarrollo sostenible. En: Revista Electrónica de Enseñanza de las Ciencias. Vol. 4(1). pp. 1-17. Consultado el 9 de marzo del 2012. Desde: http://www.saum.uvigo.es/reec.

- Vygotsky, L. S. (1995); Pensamiento y lenguaje. Madrid: Paidós., p. 237

- Von Glasersfeld, E. (1990). Introducción al constructivismo radical., pp. 20-37. En: Watzlawick P. (Edi.). La realidad Inventada. Barcelona: Gedisa. p.288.

- Weissmann, P. (2007). El papel de la escuela en el desarrollo de los procesos cognitivos. En: Revista Iberoamericana de Educación. Número 43. pp. 3-25.

- Wenstein, J.; Carrasco, E.; Negrón, B.; y Astorga, A. (2006). Sentidos de la educación y la cultura: cultivar la humanidad. Santiago de Chile: Lom. p. 263

- Ziechner, K. M. (1993). El maestro como profesional reflexivo. En: Cuadernos de Pedagogía, Número 220. pp. 44-49.

1. Docente-Investigador, (UFPS) Universidad Francisco de Paula Santander Director, Grupo de Investigación "GIRS" en Responsabilidad Social

2. La auto-plasticidad es entendida como la capacidad del cerebro de organizarse y modificarse en cualquier momento, es decir, en cualquier estado de desarrollo de la persona. 